\title{
Scavenging new insights into atherogenesis
}

\author{
Menno P.J. de Winther and Marten H. Hofker \\ Cardiovascular Research Institute Maastricht, University of Maastricht, Maastricht, The Netherlands
}

Commentary

See related articles,

pages 1049-1056

and 1095-1108.

Address correspondence to: Marten Hofker, Cardiovascular Research Institute Maastricht, University of Maastricht, PO Box 616, 6200MD Maastricht, The Netherlands. Phone: 31-43-3881420; Fax: 31-43-3884149; E-mail: hofker@lumc.nl.

According to current hypotheses, atherosclerosis begins to develop when lipoproteins accumulate in the arterial intima and become chemically modified, thus initiating local vessel wall inflammation and attracting monocytes from the circulation. Modified lipoproteins are taken up avidly by monocyte-derived macrophages, and the resulting fat-laden macrophages (now known as foam cells) reside in the vessel wall and exacerbate the local inflammatory response. Since LDL receptor-deficient (LDLR-deficient) macrophages are not attenuated in their ability to develop into foam cells, it has long been known that foam cell formation requires receptors distinct from the LDLR. Moreover, macrophages do not readily take up native plasma lipoproteins in large amounts and will only become foam cells when exposed to oxidized, acetylated, or otherwise covalently modified lipoproteins. Hence, the discovery of the "scavenger" receptors for modified lipoproteins and the elucidation of lipid-modification processes are of crucial importance in our understanding of atherogenesis.

Multiple groups, using techniques such as expression cloning and protein purification, have identified a series of scavenger receptors, shown in Table 1. At present, the known scavenger receptors (reviewed in refs. 1 and 2) comprise several classes of transmembrane receptors, all characterized by their ability to bind negatively charged macromolecules, such as modified LDL. With regard to atherosclerosis, the most interesting receptors include scavenger receptor class A (SR-A), types I and II, as well as the class-B scavenger receptors, CD36 and SR-BI.

The first scavenger receptor to be cloned was SR-A $(3,4)$, which binds with high affinity to lipoproteins that are severely modified, either by acetylation or oxidation. CD36, also called FAT for fatty acid translocase, was cloned initially as a receptor with specific affinity for oxidatively modified lipoproteins (5). Initial studies on CD36 focused on its role in the uptake of fatty acids by a variety of cells, but recent findings regarding the regulation of CD36 during monocyte activation make this receptor a strong candidate for mediating foam cell formation (6), and the paper by Febbraio et al. (7) in this issue of the JCI now demonstrates unambiguously its involvement in atherogenesis. Febbraio et al. have now expanded on their earlier characterization of CD36-deficient mice as a model for atherogenesis, crossing their knockout allele (8) onto an apo E ( $a p o E)$ gene-deficient background that confers spontaneous atherogenesis in mice. Absence of CD36, they find, strongly reduces atherosclerotic development in this genetic background. After 12 weeks on a Western-type diet, aortic trees from CD36-apoE double-knockout mice show a $76 \%$ reduction in lesional area, as compared with apoE knockout mice, and lesional area in the aortic sinus is reduced by $45 \%$. Consistent with these in vivo data, Febbraio and coworkers also show that, in the presence of different forms of modified LDL, foam cell formation is strongly reduced in vitro. In addition, they identify CD36 as the receptor for myeloperoxidase-modified (MPO-modified) lipoproteins. MPO has been demonstrated previous- ly to convert LDL into atherogenic particles that strongly induce foam cell formation in vitro.

Scavenger receptor knockouts and the importance of genetic background. During the past few years, the role of SR-A in atherosclerosis has been tested extensively. In 1997 Suzuki et al. published the generation and first analysis of SRA-deficient mice (9). On an apoE-deficient background, SR-A-deficient mice showed a $60 \%$ reduction in atherosclerotic lesion development, suggesting a strong proatherogenic role for the SRA. However, this idea has been difficult to generalize in other mouse models. Thus, when it occurs on an LDLR-deficient background, absence of SR-A results in only a $20 \%$ reduction in atherosclerosis (10). The APOE3-Leiden mouse, which has also been used as an atherogenic model, carries a dominantnegative human $A P O E$ variant as transgene and exhibits hyperlipidemia and susceptibility to diet-induced atherosclerosis. Nevertheless, no decrease in atherosclerosis is observed when SRA-deficient mice are crossed with APOE3-Leiden mice (11).

These results demonstrate that a proor antiatherogenic role of SR-A depends on the mouse model used to provide the atherogenic background, and they highlight the complex roles of apo $\mathrm{E}$ in pathogenesis. Apo $\mathrm{E}$ acts not only in plasma lipoprotein metabolism,

\section{Table 1}

Macrophage scavenger receptors and lipid-modifying enzymes involved in atherogenesis

\begin{tabular}{|c|c|c|c|c|}
\hline Receptors & \multicolumn{2}{|l|}{ Major ligands } & $\begin{array}{l}\text { Pro/anti-atherogenic } \\
\text { in animal model }\end{array}$ & Reference \\
\hline SR-A & \multirow{2}{*}{\multicolumn{2}{|c|}{$\begin{array}{l}\text { acetyl LDL, oxidized LDL } \\
\text { oxidized LDL }\end{array}$}} & pro/anti & $(9-1$ \\
\hline CD36 & & pro & ( \\
\hline SR-BI & & & anti & $(16,2$ \\
\hline CD68 & \multicolumn{2}{|l|}{ oxidized LDL? } & ND & \\
\hline Enzymes & Modifies through & Target & & \\
\hline Myeloperoxidase & nitrogen species & protein & ND & \\
\hline Lipoxygenase & oxygenation & $\mathrm{PL}, \mathrm{CE}$ & pro & \\
\hline Secretory phospholipase $A_{2}$ & hydrolysis & $\mathrm{PL}$ & pro & \\
\hline Sphingomyelinase & hydrolysis & PL & ND & \\
\hline
\end{tabular}

PL, phospholipids; CE, cholesterylesters; ND, no data. 


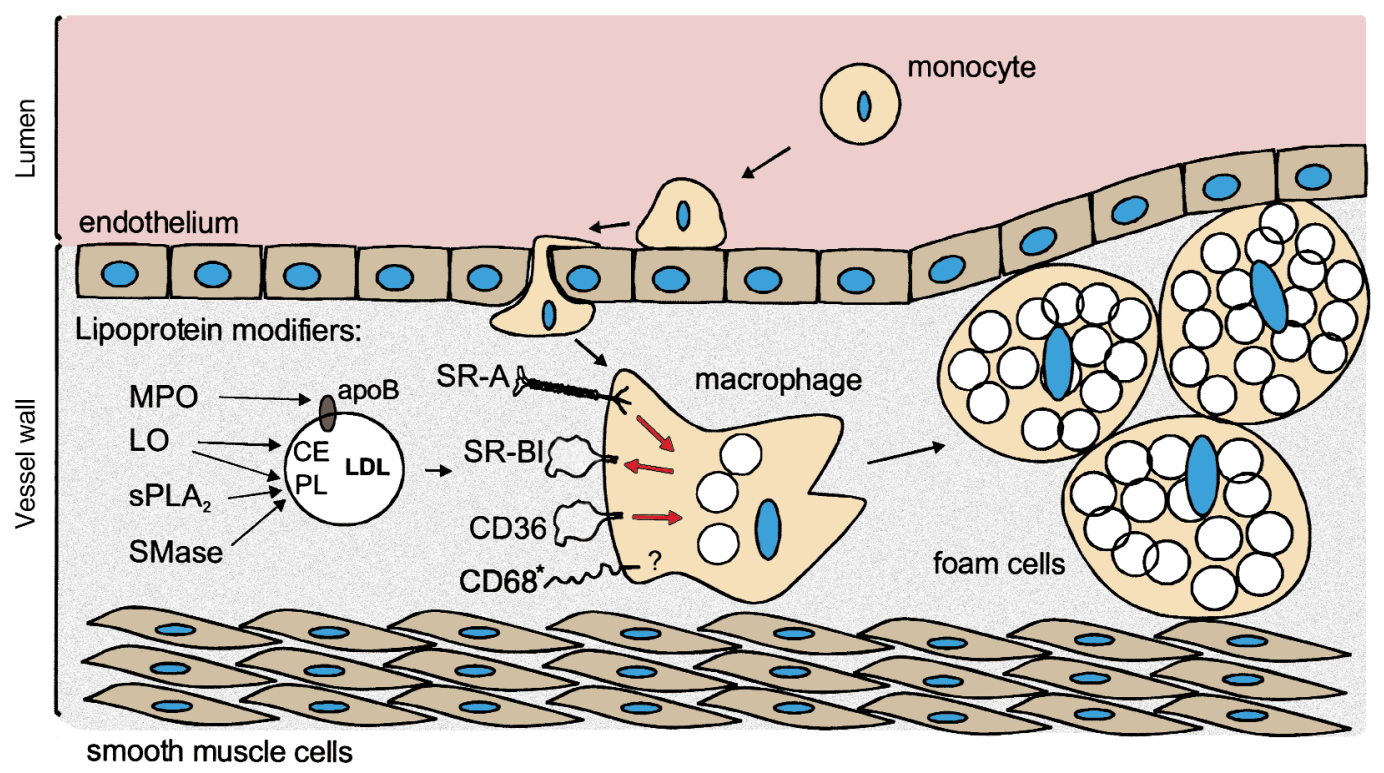

\section{Figure 1}

Lipid-modifying enzymes and macrophage scavenger receptors that may affect foam cell formation and atherogenesis. From left to right: putative lipoprotein modifiers, macrophage scavenger receptors, and fat-laden foam cells. Arrows from the lipoprotein-modifying enzymes indicate the components of lipoproteins they can act on. Red arrows within the macrophage indicate whether a given scavenger receptor mediates cholesterol uptake or efflux. Circles in macrophages indicate intracellularly accumulated cholesteryl esters. CD68 is included in this figure because it has been shown to bind oxidized LDL, but its role in modified LDL uptake is not yet clear. SR-A, scavenger receptor class A type I/II (9-11); SR-BI, scavenger receptor class B type I (16, 24). MPO, myeloperoxidase; 12/15-LO, lipoxygenase (25); sPLA2, secretory phospholipase $A_{2}$ (26); SMase, sphingomyelinase; CE, cholesteryl ester; PL, phospholipids.

but also in the efflux of cholesterol from macrophages, a process that directly opposes foam cell formation. This dual role of apo $\mathrm{E}$ has been elegantly demonstrated using bone marrow transplantation. When Fazio et al. (12) reconstituted wild-type mice with apo E-deficient bone marrow, they showed that lesions developed rapidly despite normal plasma lipid levels (12). In addition, apo $E$ mediates the binding of lipoproteins to extracellular proteoglycans and, therefore, to the vessel wall, and the loss of vascular adhesion may well alter the susceptibility of lipoproteins to modification or to uptake by macrophages (13). Both of these considerations could affect the relative contribution of SR-A to atherogenesis and may help account for the divergent findings in different genetic backgrounds. This complexity underscores the importance of studying the effects of such a mutation in a variety of mouse models. Fortunately, there are many atherogenic models available from which to choose (14).

Another scavenger receptor closely related to CD36 is SR-BI. This receptor binds specifically to HDL particles and mediates selective lipid uptake (reviewed in ref. 15). Using adenovirus-mediated overexpression of SR-BI and a SR-BIdeficient mouse model, Rigotti et al. have shown that this receptor participates in reverse cholesterol transport. In addition to HDL, however, SR-BI also binds native and modified LDL and mediates cholesterol flow from cells to HDL. Recently, Trigatti et al. described doubly deficient mice lacking SR-BI and apo E, which show an increase in lesion development relative to apo E-deficient mice (16). So far, no direct evidence has been published showing a direct role of SR-BI in mediating modified LDL uptake and foam cell formation in macrophages. Trigatti et al. propose that accelerated atherosclerosis arises in their double-knockout model either from increased levels of the atherogenic VLDL or from reduction in SRBI-mediated cholesterol efflux from the vessel wall or other changes in reverse cholesterol transport.

Lipoprotein modification in vitro and in vivo. The evidence is overwhelming that modified LDL promotes atherosclerosis (17), but it has been less clear precisely which modifications and which scavenger receptors are most important in this disease pathway. Although a plethora of excellent in vitro studies have been performed, the relevance for the mechanisms of lipoprotein modification occurring in vivo is poorly established, and functional studies are just beginning to appear. The exact mechanism has been difficult to establish, in part because lesions contain a wide range of modified LDL particles. As shown in Figure 1, LDL can be modified through the action of a broad range of enzymes, including sphingomyelinase (18) and phospholipase $\mathrm{A}_{2}$ (19), which convert native phospholipids in LDL to more biologically active phospholipids. Secreted phospholipase $\mathrm{A}_{2}$ also makes LDL more susceptible to modification by other enzymes, and sphingomyelinase causes LDL aggregation. In addition, other possibly relevant modifying enzymes are less specific for phospholipids. These include lipoxygenase, locally expressed nitric oxide synthase and MPO, which converts $L D L$ into an atherogenic form in vitro that can induce foam cell formation in macrophages (20). Until recently, the uptake route for MPO-modified LDL was unknown, but this issue of the $J C I$ also features work from Podrez et al. (21), which, together with the report by Febbraio et al. (7), convincingly identifies CD36 as the major receptor for uptake of MPO-modified LDL. As 
Podrez et al. show (21), absence of CD36 reduces uptake of MPO-modified LDL by almost $90 \%$ and reduces foam cell formation by more than half. Managing atherosclerosis. Considering the crucial role of CD36 in foam cell formation, what can be done to prevent atherosclerosis? Drugs that systemically block CD36 may not work as desired. The multiple role of CD36 as scavenger receptor, fatty acid transporter, and mediator of apoptotic cell uptake implies that these processes may all become affected by such a drug. Furthermore, the effect of CD36 deficiency in humans has emerged from genetic studies and argues against such an approach. In Japan, 2-3\% of the general population is deficient for CD36, but, interestingly, $12 \%$ of Japanese patients with coronary heart disease (CHD) carry this deficiency (22). Hence, loss of CD36 function is associated with cardiovascular diseases, and interference with CD36 function appears more likely to cause than to inhibit CHD. A more promising approach to prevent foam cell formation may be the local delivery of drugs to the vessel wall or the reduction of CD36 ligands, either by reducing cholesterol levels or by targeting the MPO system specifically.

The antiatherogenic role of CD36 still requires rigorous testing in different animal models. Despite the clear pathological role of foam cell formation under conditions of severe or prolonged hyperlipidemia, it remains plausible that uptake of modified lipoproteins by macrophages is beneficial in other circumstances, possibly including milder hyperlipidemia (23). In light of this possibility, more extensive studies are needed to determine the physiological con- sequences of therapies that block the function of CD36 or other scavenger receptors. The establishment of CD36 as an important scavenger receptor and the identification of MPO-modified lipoproteins as its main ligand represents a major advance toward this end.

\section{Acknowledgments}

The authors thank C. Wijmenga for critical reading of the manuscript.

1. Greaves, D.R., Gough, P.J., and Gordon, S. 1998. Recent progress in defining the role of scavenger receptors in lipid transport, atherosclerosis and host defence. Curr. Opin. Lipidol. 9:425-432.

2. Steinbrecher, U.P. 1999. Receptors for oxidized low density lipoprotein. Biochim. Biophys. Acta. 1436:279-298.

3. Kodama, T., Reddy, P., Kishimoto, C., and Krieger, M. 1988. Purification and characterization of a bovine acteyl low density lipoprotein receptor. Proc. Natl. Acad. Sci. USA. 85:9238-9242.

4. Freeman, M., et al. 1990. An ancient, highly conserved family of cysteine-rich protein domains revealed by cloning type I and type II murine macrophage scavenger receptors. Proc. Natl. Acad. Sci. USA. 87:8810-8814.

5. Endemann, G., et al. 1993. CD36 is a receptor for oxidized low density lipoprotein. J. Biol. Chem. 268:11811-11816.

6. Nakata, A., et al. 1999. CD36, a novel receptor for oxidized low-density lipoproteins, is highly expressed on lipid-laden macrophages in human atherosclerotic aorta. Arterioscler. Thromb. Vasc. Biol. 19:1333-1339.

7. Febbraio, M., et al. 2000. Targeted disruption of the class B scavenger receptor, CD36, protects against atherosclerotic lesion development in mice. J. Clin. Invest. 105:1049-1056.

8. Febbraio, M., et al. 1999. A null mutation in murine CD36 reveals an important role in fatty acid and lipoprotein metabolism. J. Biol. Chem. 274:19055-19062.

9. Suzuki, H., et al. 1997. A role for the macrophage scavenger receptors in atherosclerosis and susceptibility to infection. Nature. 386:292-296.

10. Sakaguchi, H., et al. 1998. Role of macrophage scavenger receptors in diet-induced atherosclerosis in mice. Lab. Invest. 78:423-434.

11. De Winther, M.P.J., et al. 1999. Scavenger receptor deficiency leads to more complex atherosclerotic lesions in APOE3Leiden transgenic mice. Atherosclerosis. 144:315-321.
12. Fazio, S., et al. 1997. Increased atherosclerosis in mice reconstituted with apolipoprotein E null macrophages. Proc. Natl. Acad. Sci. USA. 94:4647-4652.

13. Ji, Z.S., Pitas, R.E., and Mahley, R.W. 1998. Differential cellular accumulation/retention of apolipoprotein E mediated by cell surface heparan sulfate proteoglycans. Apolipoproteins E3 and E2 greater than e4. J. Biol. Chem. 273:13452-13460.

14. Breslow, J.L. 1996. Mouse models of atherosclerosis. Science. 272:685-688.

15. Rigotti, A., et al. 1997. Scavenger receptor BI: a cell surface receptor for high density lipoprotein. Curr. Opin. Lipidol. 8:181-188.

16. Trigatti, B., et al. 1999. Influence of the high density lipoprotein receptor SR-BI on reproductive and cardiovascular pathophysiology. Proc. Natl. Acad. Sci. USA. 96:9322-9327.

17. Steinberg, D. 1997. Low density lipoprotein oxidation and its pathobiological significance. J. Biol. Chem. 272:20963-20966.

18. Xu, X.X., and Tabas, I. 1991. Sphingomyelinase enhances low density lipoprotein uptake and ability to induce cholesteryl ester accumulation in macrophages. J. Biol. Chem. 266:24849-24858.

19. Leitinger, N., et al. 1999. Role of group II secretory phospholipase A2 in atherosclerosis. 2. Potential involvement of biologically active oxidized phospholipids. Arterioscler. Thromb. Vasc. Biol. 19:1291-1298.

20. Podrez, E.A., Schmitt, D., Hoff, H.F., and Hazen, S.L. 1999. Myeloperoxidase-generated reactive nitrogen species convert LDL into an atherogenic form in vitro. J. Clin. Invest. 103:1547-1560.

21. Podrez, E.A., et al. 2000. Macrophage scavenger receptor $\mathrm{CD} 36$ is the major receptor for $\mathrm{LDL}$ modified by monocyte-generated reactive nitrogen species. J. Clin. Invest. 105:1095-1108.

22. Watanabe, K., et al. 1997. Different patterns of 123I-BMIPP myocardial accumulation in patients with type I and II CD36 deficiency. Kaku Igaku. 34:1125-1130.

23. Ross, R. 1999. Atherosclerosis: an inflammatory disease. N. Engl. J. Med. 340:115-126.

24. Arai, T., Wang, N., Bezouevski, M., Welch, C., and Tall, A.R. 1999. Decreased atherosclerosis in heterozygous low density lipoprotein receptor-deficient mice expressing the scavenger receptor BI transgene. J. Biol. Chem. 274:2366-2371.

25. Cyrus, T., et al. 1999. Disruption of the $12 / 15$ lipoxygenase gene diminishes atherosclerosis in apo E-deficient mice. J. Clin. Invest. 103:1597-1604.

26. Ivandic, B., et al. 1999. Role of group II secretory phospholipase A2 in atherosclerosis. 1. Increased atherogenesis and altered lipoproteins in transgenic mice expressing group IIa phospholipase A2. Arterioscler. Thromb. Vasc. Biol. 19:1284-1290. 